\title{
Moral Sharing: Teilen zwischen Unternehmen und Konsumenten
}

Die folgende Studie untersucht ein alternatives Sharing Modell, in welchem durch eine nicht-monetäre Kooperation zwischen Einzelhandel und Konsumenten innovative Wege beschritten werden, um der Lebensmittelverschwendung zu begegnen. Mittels einer empirischen Studie werden Motivationen von Einzelhandel und Konsumenten beleuchtet und Implikationen für CSR Programme gegeben.

Johanna F. Gollnhofer 
Die Sharing Economy wird derzeit als wirtschaftliches Zukunftsmodell thematisiert (Botsman/Rogers 2011). Bereits 1984 stellte Martin Weitzmann fest, dass sich der Wohlstand erhöht, je mehr unter den Marktteilnehmern geteilt wird (Weitzman 1986). 2013 griff die Cebit den Gedanken auf und machte es zu ihrem Leitthema. Aufgrund von unterschiedlichen Motivationen, wie zum Beispiel finanziellen, ökologischen oder pro-sozialen Gesichtspunkten, sind mannigfaltige Marktstrukturen und Geschäftsmodelle entstanden. Mittels einer Neudefinierung des wirtschaftlichen Wertes von Objekten und der Ausnutzung von Überhangkapazitäten werden Konsumgüter länger und/oder intensiver genutzt (O’Brien 1999). Die prominenten Sharing-Modelle lassen sich anhand der Achsen monetäre/nicht-monetäre und CtoC (Consumer-to-Consumer)/BtoC (Business-to-Consumer) einordnen (vgl. Tabelle 1). Die erste Achse bezieht sich darauf, ob bei dem Akt des Sharings monetäre Mittel fließen, während die zweite Achse angibt, zwischen welchen Parteien das Sharing stattfindet.

Zum einen spricht man von „Pseudo Sharing“ (Belk 2014), das heißt von kapitalistischen Modellen, welche unter dem Deckmantel des pro-sozialen Sharing-Gedankens operieren, jedoch neue, kreative Geschäftsmodelle darstellen (z.B. DriveNow von BMW). In diesem Sinne wird Sharing oftmals als ein neuer Ausdruck für „ein Auto ausleihen“ verwendet (Fournier/Eckhardt/Haddad 2013). Diese Sharing-Modelle können im oberen rechten Quadranten von Tabelle 1 eingeordnet werden.

Auch Konsumenten praktizieren eine monetäre Form des Sharings, indem sie untereinander ihre (temporär) nicht genutzten Ressourcen, wie beispielsweise Autos oder sonstige Gegenstände wie Bohrmaschinen, gegen Geld oder Gegenleistungen auf dem Markt (z.B. www.leihdirwas.de) tauschen.

Der pro-soziale Gedanke des Sharings spiegelt sich in Ideen wie Couchsurfing oder Buchtauschkreisen wider, welche ohne finanzielle Gegenleistung oder Kooperation mit Unternehmen operieren (Albinsson/Perera 2012; Corciolani/Dalli 2014).

Während nicht-monetäre Sharing-Modelle zwischen Konsumenten vielfach theoretisch konzeptualisiert worden sind (Bardhi/Eckhardt 2012; Arnould/Rose 2014; Corciolani/Dalli 2014), ist die Natur von nicht-monetären Sharing-Modellen auf BtoC-Ebene um ein Vielfaches unklarer. Ein Grund dafür ist, dass diese Art von Sharing nicht mit dem Grundverständ-

\section{Tab. 1 Beispiele aus der Sharing Economy}

\begin{tabular}{|l|l|l|}
\hline \multicolumn{1}{|c|}{ CtoC } & BtoC \\
\hline Monetär & $\begin{array}{l}\text { www.leih-ein-Buch.de } \\
\text { www.leihdirwas.de } \\
\text { www.nachbarschaftsauto.de } \\
\text { www.mitfahrzentrale.de }\end{array}$ & $\begin{array}{l}\text { www.drive-now.com } \\
\text { www.callabike-interaktiv.de }\end{array}$ \\
\hline Nicht-monetär & $\begin{array}{l}\text { www.couchsurfing.com } \\
\text { www.bookcrossing.de }\end{array}$ & www.foodsharing.de \\
\hline Quelle: eigene Darstellung & \\
\hline
\end{tabular}

\section{Johanna Franziska Gollnhofer} ist wissenschaftliche Mitarbeiterin an der Forschungsstelle für Customer Insight (FCI), Universität St. Gallen, http://fci.unisg.ch/
Johanna Franziska Gollnhofer
Universität St. Gallen, St. Gallen, Schweiz
E-Mail: Johanna.gollnhofer@unisg.ch 
nis auf dem kapitalistischen Markt, auf welchen Konsumenten und Unternehmen über monetäre Transaktionen verbunden sind, übereinstimmt. Der folgende Beitrag beleuchtet die Motivation für solche Kooperationen. Ein vertieftes Verständnis von nicht-monetären Sharing-Modellen ermöglicht Unternehmen, nicht mit Reaktanz zu reagieren, sondern mit Kooperation (Belk 2014b).

\section{Teilen in der Sharing Economy}

Einzelhandel und Konsument stehen in der Regel über monetäre Beziehungen miteinander in Verbindung. Im klassischen Sinne wird zwischen produzierender und konsumierender Einheit unterschieden, welche respektive konträre Zielsetzungen, wie die Erhöhung des Gewinns und die Senkung der Ausgaben, verfolgen (Lévi-Strauss 2008; Prahalad/ Ramaswamy 2004). Aufgrund dieser dualen Struktur und einer stark firm-zentrierten Perspektive im Marketing sowie im Managementbereich ist eine Zusammenarbeit, welche nicht auf monetären Transaktionen basiert, zwischen diesen beiden Marktakteuren schwer vorstellbar. In der postmodernen Perspektive wird diese strikte Unterscheidung jedoch aufgeweicht (Firat/Venkatesh 1995), was zu einem Dialog, erhöhter Transparenz und Zugang zu Informationen zwischen verschiedenen Marktteilnehmern führt (Prahalad/Ramaswamy 2004). Der Konsument wird als befähigt, ermächtigt und aktiv wahrgenommen (Giesler/Veresiu 2014; Kozinets/Handelman 2004), was zu neuen Interaktionen und Geschäftsmodellen zwischen Konsument und Produzent, wie wir sie auch aus der Sharing Economy kennen, führt. Zum Beispiel wird die Markenidentität durch Konsumenten aktiv mitgestaltet (Holt

\section{Zusammenfassung}

1. Im Rahmen der Sharing Economy können Einzelhandel und Konsumenten auf einer nicht-monetären Basis zusammenarbeiten, um ein gemeinsames Ziel zu verfolgen.

2. In dem Fall von Foodsharing schaffen sie einen moralischen Sharing-Markt, welcher als Ziel die Eindämmung der Lebensmittelverschwendung verfolgt.

3. Dieses moralische Handeln kann über den Hebel von Corporate Social Responsibility (CSR)-Programmen zur Stärkung der Marke verwendet werden.
2002; Muniz/O’Guinn 2001), der Konsument wird oftmalsohne finanziellen Ausgleich aktiv in die Erstellung eines Produktes oder Services miteingebunden (Humphreys/Grayson 2008; Prahalad/Ramaswamy 2004) oder schafft sich aktiv einen Markt für seine Bedürfnisse (Martin/Schouten 2014). Der Konsument wird zum „Ko-Konsumenten“ oder „Prosumer“ und verwischt somit die klassischen Grenzen zwischen Konsument und Produzent (Ritzer/Dean/Jurgenson 2012). Diese Perspektive liegt aus theoretischer Sicht insbesondere Pseudo-Sharing-Modellen zugrunde, jedoch erklärt sie kaum nicht-monetäre Kooperationen zwischen Unternehmen und Konsumenten.

\section{Das Konzept von Foodsharing}

Nicht-monetäre Kooperationen zwischen Unternehmen und Konsumenten erfolgen oftmals im karitativen Bereich, wie zum Beispiel bei der Tafel, welche vom Einzelhandel erhaltene Lebensmittel an bedürftige Menschen verteilt (Hill/Stamey 1990; Riches 2002). Foodsharing nimmt eine ähnliche Rolle wie die Tafel ein, indem es aussortierte, unverkäufliche Lebensmittel an Konsumenten weiterverteilt. Diese Weiterverteilung erfolgt jedoch nicht basierend auf dem Kriterium der Bedürftigkeit; stattdessen kann jeder interessierte Konsument von diesen Lebensmitteln profitieren. Konkret gibt der Einzelhandel (z.B. Supermärkte oder Bäckereien) kostenlos Lebensmittel an Konsumenten ab (vgl. Abbildung 1). Konsumenten haben folglich die Möglichkeit, Lebensmittel kostenlos zu beziehen, anstatt diese in einem Supermarkt einzukaufen, obwohl sie sich dies aus finanzieller Sicht leisten könnten. Der eingetragene Verein Foodsharing beabsichtigt durch die Weitergabe von genießbaren Lebensmitteln, die jedoch beispielsweise nicht dem persönlichen Geschmack, kulturellen ästhetischen Ansprüchen oder gesetzlichen Richtlinien (z.B. dem Mindesthaltbarkeitsdatum) entsprechen, die Abfallmenge zu reduzieren. Foodsharing e. V. greift hierbei ausschließlich auf ehrenamtliche Mitarbeiter zurück und verfolgt keinen wirtschaftlichen Zweck, lediglich einen Idealzweck. Hiermit stellt Foodsharing einen interessanten Fall dar, in welchem Konsumenten und Unternehmen auf nicht monetärer Basis miteinander kooperieren (McGranenov 2014).

Im Laufe des zweijährigen Bestehens von Foodsharing konnten somit $1.548 .192 \mathrm{~kg}$ Lebensmittel durch den Einsatz von 7.118 Konsumenten in Kooperation mit 1.713 teilnehmenden Unternehmen gerettet werden (www.foodsharing.de/ statistik, Stand: 28. Mai 2015). Um ein tieferes Verständnis für das Zustandekommen dieser Kooperation zu gewinnen, wer- 
den im Folgenden die Motivationen von teilnehmenden Konsumenten und Betrieben untersucht.

\section{Studie und Datenanalyse}

Die zugrunde liegenden ethnografischen Daten dieser empirischen Studie wurden im Zeitraum von September 2013 bis Februar 2015 auf Grundlage eines theoretischen Samplings gesammelt. Die 18 Tiefeninterviews umfassen Gespräche mit führenden Personen bei Foodsharing e. V., (Marketing-)Managern von kooperierenden Unternehmen und bei Foodsharing aktiven Konsumenten (im Folgenden als „Foodsaver“ bezeichnet). Diese Daten werden ergänzt durch beobachtende Teilnahme sowie durch Online-Daten (Blogeinträge, Zeitungsartikel, Foodsharing Forum). Durch die Methode des permanenten Vergleichs, das heißt durch die fortwährende Verankerung der Interpretation in den Daten wird sichergestellt, dass die Ergebnisse stets einen Bezug zur Empirie aufweisen (Czarniawska 2014; Glaser/Strauss 2009; Spiggle 1994). Mittels offenem und axialem Kodieren wurden unterschiedliche Kategorien identifiziert (Flick 2009). Insgesamt wurde bei der Auswertung der Daten der Standardprozedur von ethnografischen Daten im Konsumentenverhalten gefolgt (z.B. Cayla/Arnould 2013).

Die in der folgenden Studie dargestellten Ergebnisse stellen nur einen kleinen Ausschnitt aus einem übergeordneten Forschungsprojekt dar, welches sich mit der Konzeptualisierung von alternativen Märkten beschäftigt.

\section{Ergebnisse der empirischen Studie}

In den klassischen Verkäufer-Käufer-Rollen verfolgen Einzelhändler und Konsumenten kontroverse Ziele; der Erstere strebt nach Umsatzmaximierung, der Letztere nach einer Senkung der Ausgaben für Konsumgüter. Der Einzelhandel sowie der Konsument werden als rationale Akteure dargestellt (Baudrillard 1998), welche hauptsächlich an der monetären Optimierung von Transaktionen interessiert sind. Die Ergebnisse der empirischen Studie stellen jedoch dar, wie moralische Beweggründe zu einer nicht-monetären Kooperation zwischen Einzelhandel und Konsument führen. Im Gegensatz zu anderen Modellen in der Sharing Economy, wo die Motivationsgründe oftmals primär im finanziellen Bereich (z.B. Carsharing) oder im sozialen Bereich (z.B. Couchsurfing) zu finden sind, werden bei Foodsharing auf Konsumenten- wie auch auf Unternehmensseite ökologische, das heißt moralische Gründe angeführt.

\section{Konsumenten und Foodsharing}

Wie anfangs erläutert, sind die Motivationen für eine aktive Teilnahme an der Sharing Economy mannigfaltig. Ähnlich wie bei Carsharing-Nutzern (Bardhi/Eckhardt 2012) sind die Beweggründe für Foodsharing teilweise ökonomischer Natur: Foodsaver, welche ca. zwei- bis dreimal die Woche bei kooperierenden Unternehmen abholen, geben kaum sonstige Ausgaben für Lebensmittel an. Auch soziale Gründe spielen eine Rolle, da sich Foodsharing-Treffen und Events zur Stärkung

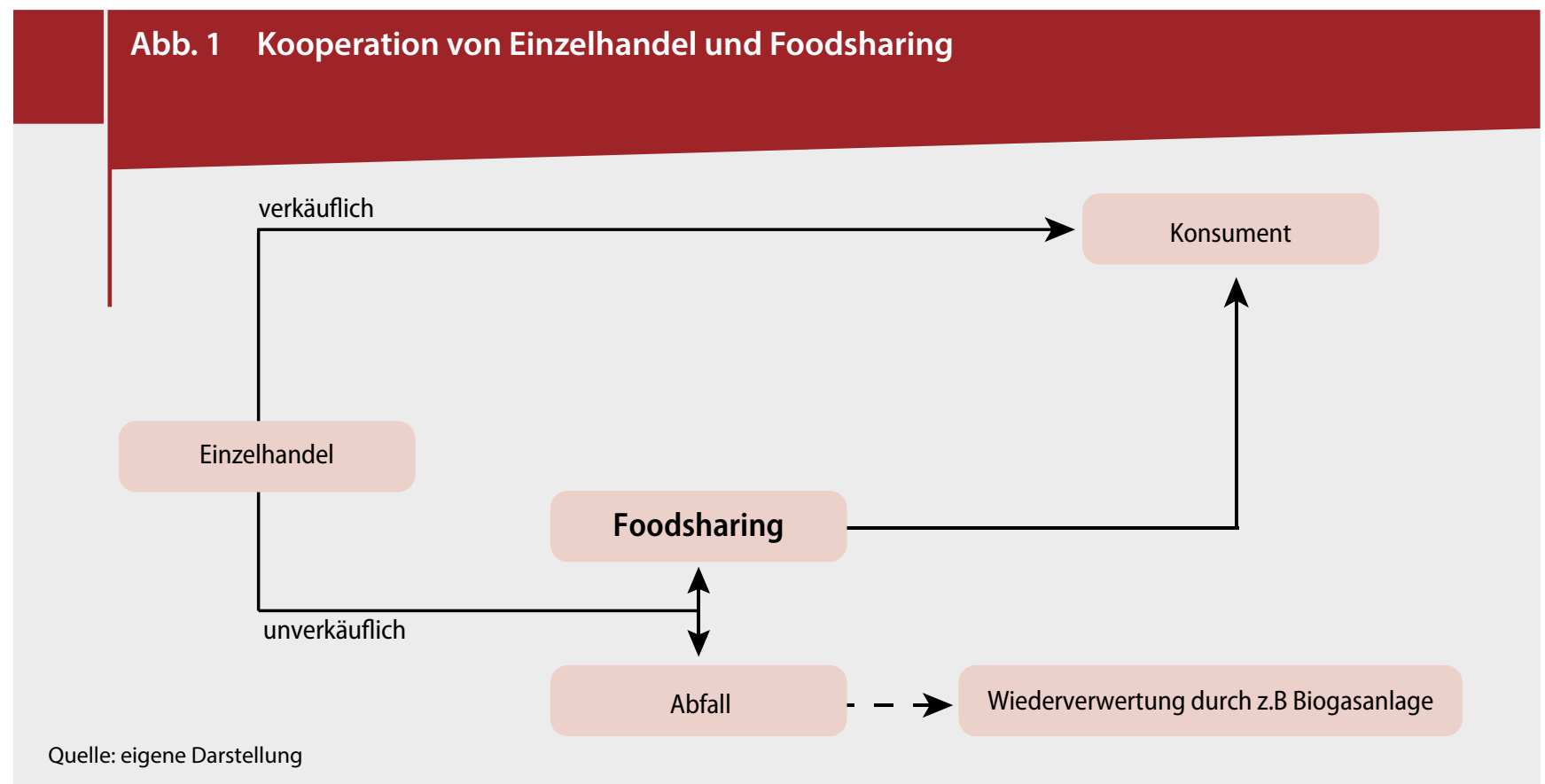


des Gemeinschaftsgefühls und des Zusammenhalts anbieten. Dennoch scheinen Gedanken bezüglich Nachhaltigkeit und Lebensmittelverschwendung der Hauptantriebspunkt zu sein. Die Motivation, bei Foodsharing mitzumachen, ist folglich ähnlich wie bei anderen Sharing-Modellen eine Mischung aus monetären, sozialen und ökologischen Beweggründen, wobei ein starker Fokus auf ökologischen Beweggründen liegt.

\section{Einzelhandel und Foodsharing}

Unternehmen nutzen die Sharing Economy größtenteils für das Generieren von Umsätzen durch die Nutzung neuer Businessmodelle (z.B. Carsharing). Ihre Motivation ist hierbei finanzieller Natur. Foodsharing versucht, Unternehmen mittels finanzieller Argumente für eine Kooperation zu gewinnen (z.B. Einsparung von Müllkosten). Die Informanten dieser Studie gaben jedoch einstimmig an, dass die Einsparung an Müllkosten marginal oder negativ ist, da gewisse Lebensmittelabfälle in Biogasanlagen gegen Geld wiederverwertet werden können.

Für teilnehmende Unternehmen steht die moralische Seite im Vordergrund, das heißt, es geht darum, Ressourcen effektiv zu verwenden, anstatt sie zu verschwenden. Mit dieser moralischen Argumentation will man dem Wert der Lebensmittel gerecht werden, die Mitarbeiter hinsichtlich der Lebensmittelverschwendung sensibilisieren und eine Vorreiterrolle in der Gesellschaft spielen. Manche Unternehmen wären sogar bereit, einen geringen Umsatzeinbruch hinzunehmen, dennoch muss ein Kompromiss zwischen Moralität und be- triebswirtschaftlichen Zielen gefunden werden. Die Angst vor Umsatzrückgängen konnte nicht bestätigt werden, was die Informanten dieser Studie darauf zurückführen, dass die Lebensmittel, die vom Einzelhandel an Foodsharing weitergegebn werden, über zentrale Verteilstellen in der jeweiligen Stadt an Individuen verteilt werden, die oftmals nicht Teil ihrer Kundschaft sind.

Die moralische Seite spiegelt sich auch in der Kooperation vieler Einzelhändler mit der Tafel wider. Foodsharing möchte jedoch nicht als Konkurrenz zur Tafel verstanden werden, sondern vielmehr als Ergänzungsangebot. Durch einen Haftungsausschluss, welcher die kooperierenden Unternehmen aus der Haftung nimmt, kann Foodsharing im Gegensatz zur Tafel auch angerissene Packungen, Kosmetika und Produkte nach dem Mindesthaltbarkeitsdatum mitnehmen und weiterverteilen. Zudem ist es Foodsharing über lokale Teams möglich, flexibel und an jedem Tag der Woche abzuholen (der Samstag kann oftmals nicht über karitative Einrichtungen abgedeckt werden).

Insgesamt richtet sich Foodsharing bei der Abholung vollkommen nach dem kooperierenden Unternehmen: Dieses kann die Abholtage und -zeiten festlegen und bei der Auswahl eines festen Teams mitwirken, welches über ein Ausweissystem verifiziert wird. Die Ergebnisse der vorliegenden Studie zeigen, dass die Professionalität und Flexibilität in der Organisation von Foodsharing dazu geführt haben, dass sich Einzelhändler mancherorts dazu entschieden haben, ausschließlich mit Foodsharing anstatt mit der Tafel zu kooperieren.

\section{Tab. 2 Beispiele für Initiativen gegen Lebensmittelverschwendung}

\begin{tabular}{|c|c|}
\hline Ansätze & Beispiele \\
\hline $\begin{array}{l}\text { Verkauf von Obst und Gemüse mit Schönheitsfehlern zu einem vergünstigten } \\
\text { Preis, welche normalerweise nicht den Weg in den Einzelhandel finden }\end{array}$ & $\begin{array}{l}\text { - "Unique“ (Coop 2013) } \\
\text { - "Inglorious Fruits" (Intermarché 2014) } \\
\text { - "Keiner ist perfekt“ (Edeka 2014) }\end{array}$ \\
\hline $\begin{array}{l}\text { Offenlegung der Abfallzahlen für die Sensibilisierung des Konsumenten hin- } \\
\text { sichtlich des Kaufverhaltens }\end{array}$ & - M\&S, Waitrose, Co-op, Tesco (in UK) \\
\hline $\begin{array}{l}\text { Verkauf von Produkten, welche über traditionelle Wege keinen Absatz finden, } \\
\text { wegen z. B. Mindesthaltbarkeitsdatum oder angerissenen Verpackungen }\end{array}$ & $\begin{array}{l}\text { - www.biorampe.ch } \\
\text { - www.aess-bar.ch } \\
\text { - www.approvedfood.co.uk }\end{array}$ \\
\hline Verkauf ohne Verpackung & - www.orginal-unverpackt.de \\
\hline $\begin{array}{l}\text { Restaurants, welche mit unverkäuflichen Lebensmitteln oder Lebensmitteln, } \\
\text { die nicht den ästhetischen Standards entsprechen, kochen }\end{array}$ & $\begin{array}{l}\text { - www.culinarymisfits.de } \\
\text { - www.dinnerexchangeberlin.com }\end{array}$ \\
\hline Sonstige Unterstützung für Foodsharing.de & $\begin{array}{l}\text { - Bereitstellung von Kühlschränken für die Verteil- } \\
\text { punkte für die Lebensmittel: Lemonaid (www. } \\
\text { lemon-aid.de) } \\
\text { - Rechtliche Unterstützung: www.abd-partner.de }\end{array}$ \\
\hline
\end{tabular}

Quelle: eigene Darstellung 


\section{Diskussion}

Modelle in der Sharing Economy nutzen auf oftmals kreative Art und Weise Überhangskapazitäten oder Ressourcen, welche im klassischen Wirtschaftskreislauf häufig ungenutzt bleiben. Konkret werden große Mengen von Lebensmitteln in Biogasanlagen zur Energieerzeugung weiterverwendet (Zhang/ElMashad/Hartman 2007). Hierbei handelt es sich jedoch um eine Art des „downcyclings“ (Steinhilper/Hieber 2001), das heißt eine Abwertung des Wertes von Lebensmitteln, da sie nicht mehr länger als Lebensmittel genutzt werden, sondern als Brennmaterial. Der Fall von Foodsharing zeigt auf, inwiefern Konsumenten und Unternehmen gemeinsam einen moralischen Markt im Raum der Sharing Economy schaffen, in welchem Lebensmittel wieder einen ideelen Wert bekommen und mehr sind als bloß eine Ware. Im Gegensatz zu karitativen Einrichtungen geht es bei der Weiterverteilung der Lebensmittel nicht um den Menschen in „Not“, sondern um den Wert des Lebensmittels. Dies geschieht durch eine Fokussierung auf ein übergeordnetes Ziel (Lusch 2006; Payne/Storbacka/Frow 2008) - ein nachhaltiger und bewusster Umgang mit Lebensmitteln -, und durch die Realisierung, dass der Wert eines Lebensmittels, beispielsweise durch das Mindesthaltbarkeitsdatum, sozial-konstruiert ist (Berger/Luckmann 1991). Dadurch können bestehende Strukturen hinterfragt, verstanden und geändert werden, was zu alternativen Modellen im Rahmen der Sharing Economy führen kann. Hiermit wird das realisiert, was Botsman und Roger (2010) als ,soziale Revolution“ beschreiben, das heißt eine Balance zwischen Eigeninteresse (finanzielle Komponente) und übergeordneten Interessen (nachhaltiger Umgang mit Lebensmitteln) bei Konsumenten wie auch bei teilnehmenden Unternehmen. Unternehmen können somit einer gesellschaftlichen und umweltbezogenen Verantwortung gerecht werden (McWilliams/Siegel 2001).

\section{Implikationen für Manager}

- Offenheit gegenüber kreativen Ansätzen, welche teilweise der kapitalistischen Marktlogik widersprechen.

Die kostenlose Weitergabe von ehemaliger Handelsware an zahlungsfähige Konsumenten erscheint auf den ersten Blick paradox. Der Fall Foodsharing zeigt auf, wie Unternehmen jedoch nicht nur aus finanziellen, sondern auch aus moralischen Gesichtspunkten an der Sharing Economy teilnehmen können. Es gilt hierbei, einen Mittelweg zwischen moralischem und betriebswirtschaftlichem Handeln zu finden.

- Moralisches und betriebswirtschaftliches Handeln sind keine Gegensätze.
Moralische und betriebswirtschaftliche Gesichtspunkte schließen sich nicht aus. Die Effekte von Foodsharing auf den langfristigen Umsatz der kooperierenden Unternehmen sind aufgrund der weiteren Entwicklung von Foodsharing hinsichtlich Einfluss, Größe und Ausmaß unsicher. Foodsharing kann jedoch genutzt werden, um gegen die Lebensmittelverschwendung vorzugehen und die eigene Marke zu stärken, da CSR als Werkzeug des strategischen Managements (McWilliams/Siegel/Wright 2006) es ermöglicht, moralische und strategische Gesichtspunkte zu vereinen.

- Foodsharing als integraler Bestandteil von CSR-Programmen. In einem reifen und gesättigten Markt wie dem Einzelhandel, welcher zunehmend von Preiskämpfen dominiert wird, ist es notwendig sich über andere Faktoren vom Wettbewerb zu differenzieren (Meijer 2005; Wühle 2007; Lai/Cheng/Tang 2010). Nachhaltiger Umgang mit Lebensmitteln ist meistens in den Corporate-Social-Responsibility-Programmen des Einzelhandels verankert und wird oftmals über Verbrennung in Biogasanlagen bewerkstelligt. Foodsharing bietet jedoch die Möglichkeit, noch genießbare Lebensmittel weiterzugeben, anstatt sie zu vernichten. Durch eine Darstellung der Kooperation mit Foodsharing in CSR-Programmen kann somit die eigene Marke gestärkt werden (Wühle 2007).

Weitere Inspirationen für einen nachhaltigen Umgang mit Lebensmitteln können der Tabelle 2 entnommen werden. Hier sind Beispiele aufgeführt, wie der Einzelhandel oder der kreative Konsument aktiv gegen Lebensmittelverschwendung vorgehen kann, sei es durch innovative Verkaufsmodelle oder durch die kostenlose Weitergabe von Lebensmitteln.

Das Konzept von Foodsharing ist auch auf andere Branchen übertragbar, welche einen hohen Grad an Verschwendung oder kurze Produktlebenszyklen aufweisen (z. B. Drogeriemärkte oder Modeindustrie). Auch hier sollten sich kreative Ansätze zur Lösung des Problems finden lassen.

\section{Kernthesen}

1. Sharing zwischen Einzelhandel und Konsument erfolgt nicht ausschließlich über monetäre Anreize.

2. Es gilt, eine Balance zwischen betriebswirtschaftlichem und moralischem Handeln zu finden.

3. Mittels CSR-Programmen kann moralisches Handeln zur Stärkung der Marke verwendet werden 


\section{Fazit}

Durch den empirischen Ansatz konnte aufgezeigt werden, wie Unternehmen und Konsumenten in einem nicht-monetären BtoC-Sharingmodell auf Grundlage von moralischen Überlegungen zusammenfinden. Insbesondere auf Unternehmensseite erscheint ein tieferes Verständnis für alternative SharingModelle unumgänglich. Erstens ermöglicht solch ein Verständnis, diesen Modellen, welche nicht in einem kapitalistischen Grundverständnis verankert sind, mit Offenheit gegenüberzutreten und im Zuge von CSR-Programm für die Stärkung der eigenen Marke zu nutzen. Zweitens wird ein tieferes Konsumentenverständnis gefördert. Beispielsweise zeigt das Exempel von Foodsharing auf, dass Konsumenten dank des Internets und weltweiter Medienberichterstattung stark für das Thema Lebensmittelverschwendung im Einzelhandel sensibilisiert worden sind.

\section{Literatur}

Albinsson, P./Perera, Y. (2012): Alternative Marketplaces in the 21st Century: Building Community through Sharing Events, in: Journal of Consumer Behaviour, 11, (4), S. 303-315.

Arnould, E./Rose, A. (2014): Mise Au Point: Du Concept de 'Partage’ À La Mutalité En 'Consumer Research, in: Revue du MAUSS, 44, (2), S. 217-228.

Bardhi, F./Eckhardt, G. (2012): Access-Based Consumption: The Case of Car Sharing, in: Journal of Consumer Research, 39, (4), S. 881-898.

Baudrillard, J. (1998): The Consumer Society: Myths and Structures, London.

Belk, R. (2014a): Sharing vs. Pseudo-Sharing in Web 2.0, in: Anthropologica, 4, (2), S. 1-47.

Belk, R. (2014b): You Are What You Can Access: Sharing and Collaborative Consumption Online, in: Journal of Business Research, 67, (8), S. 1595-1600.

Berger, P./Luckmann, T. (1991): The Social Construction of Reality, London.

Botsman, R./Rogers R. (2011): What's Mine Is Yours: How Collaborative Consumption Is Changing the Way We Live, London.

Cayla, J./Arnould, E. (2013): Ethnographic Stories for Market Learning, in: Journal of Marketing, 77, (3), S. 1-16.

Corciolani, M./Dalli, D. (2014): Gift-Giving, Sharing and Commodity Exchange at Bookcrossing.com: New Insights from a Qualitative Analysis, in: Management Decision, 52, (4), S. 755-776.

Czarniawska, B. (2014): Social Science Research - From Field to Desk, London.

Firat, A./Venkatesh, A. (1995): Liberatory Postmodernism and the Reenchantment of Consumption, in: Journal of Consumer Research, 22, (3), S. 239-267.
Flick, U. (2009): An Introduction to Qualitative Research, London.

Fournier, S./Eckhardt, G./Haddad, A. (2013): Learning to Play in the New' Share Economy, in: Harvard Business Review, 91, (7), S. 2701-2703.

Giesler, M./Veresiu, E. (2014): Creating the Responsible Consumer: Moralistic Governance Regimes and Consumer Subjectivity, in: Journal of Consumer Research, 41, (3), S. 840-857.

Glaser, B./Strauss, A. (2009): The Discovery of Grounded Theory: Strategies for Qualitative Research, New Jersey.

Hill, R./Stamey, M. (1990): The Homeless in America: An Examination of Possessions and Consumption Behaviors, in: Journal of Consumer Research, 17, (3), S. 303-220.

Holt, D. (2002): Why Do Brands Cause Trouble ? A Dialectical Theory of Consumer Culture and Branding, in: Journal of Consumer Research, 29, (1), S. 70-90.

Humphreys, A./Grayson, K. (2008): The Intersecting Roles of Consumer and Producer: A Critical Perspective on Co-production, Cocreation and Prosumption, in: Sociology Compass, 2, (3), S. 963-980.

Kozinets, R./Handelman, J. (2004): Adversaries of Consumption: Consumer Movements, Activism, and Ideology, in: Journal of Consumer Research, 31, (3), S. 691-704.

Lai, K./Cheng, T./Tang, A. (2010): Green Retailing: Factors for Success, in: California Management Review, 52, (2), S. 6-31.

Lévi-Strauss, C. (2008): Structural Anthropology, New York.

Martin, D./Schouten, J. (2014): Consumption-Driven Market Emergence, in: Journal of Consumer Research, 40, (5), S. 855-870.

McGranenov, S. (2014): Finding Takers for Lonely Leftovers in a Culinary Nook of the Sharing Economy, http://www.nytimes. com/2014/11/27/world/europe/german-matchmakers-pair-lonelyleftovers-and-rumbling-bellies.html?_r=0, Abruf 26.05.2015.

\section{Handlungsempfehlungen}

1. Moralisches und umweltbewusstes Handeln wird von Konsumenten mehr und mehr gefordert. Um betriebswirtschaftliche Ziele mit moralischen zu vereinbaren, müssen oftmals neue Wege gegangen werden.

2. Marketingmanager sollten bezüglich alternativen Sharing-Modellen gegenüber offen sein und überlegen, wie mit ihnen konstruktiv umgegangen werden kann, anstatt sie zu bekämpfen.

3. Die Erwähnung von Foodsharing im Zuge von Corporate-Social-Responsibility-Programmen trägt zur Stärkung der Marke bei. 
McWilliams, A./Siegel, D. (2001): Corporate Social Responsibility: A Theory of the Firm Perspective, in: Academy of management review, 26, (1), S. 117-127.

McWilliams, A./Siegel, D./Wright, P. (2006): Corporate Social Responsibility: Strategic Implications, in: Journal of Management Studies, 43, (1), S. 1-18.

Meijer, M.-M. (2005): Corporate Social Performance as a Bottom Line for Consumers, in: Business \& Society, 44, (4), S. 442-461.

Muniz, A./O’Guinn, T. (2001): Brand Community, in: Journal of Consumer Research, 27, (4), S. 412-432.

O'Brien, M. (1999): Rubbish Values: Reflections on the Political Economy of Waste, in: Science as Culture, 8, (3), S. 269-295.

Prahalad, C./Ramaswamy, V. (2004): Co-Creation Experiences: The next Practice in Value Creation, in: Journal of Interactive Marketing, 18, (3), S. 5-14.

Riches, G. (2002): Food Banks and Food Security: Welfare Reform, Human Rights and Social Policy. Lessons from Canada?, in: Social Policy \& Administration, 36, (6), S. 648-663.

Ritzer, G./Dean, P./Jurgenson, N. (2012): The Coming of Age of the Prosumer, in: American Behavioral Scientist, 56, (4), S. 379-398.
Spiggle, S. (1994): Analysis and Interpretation of Qualitative Data in Consumer Research, in: Journal of Consumer Research, 21, (3), S. 491-503.

Steinhilper, R./Hieber, M. (2001): Remanufacturing-the Key Solution for Transforming 'downcycling' into 'upcycling' of Electronics, in: Proceedings of the 2001 IEEE International Symposium on IEEE, S. 161-166.

Weitzman, M. (1986): The Share Economy: Conquering Stagflation, Boston.

Wühle, M. (2007): Mit CSR Zum Unternehmenserfolg - Gesellschaftliche Verantwortung Als Wertschöpfungsfaktor, Saarbrücken.

Zhang, R./El-Mashad, H./Hartman, K. (2007): Characterization of Food Waste as Feedstock for Anaerobic Digestion, in: Bioresource technology, 98, (4), S. 929-35.

\section{Springer Gabler}

\section{So werden Sie ein Top-Verkäufer}

Rainer Frieß

Mit olympischem

Verkaufen zum Erfolg

4. Aufl. 2015.

XIII, 114 S. 54 Abb. in Farbe. Brosch.

$€$ (D) $29,99 \mid €$ (A) $30,83 \mid{ }^{*} \mathrm{~s} F r 37,50$

ISBN 978-3-658-05648-3 (Print)

$€ 22,\left.99\right|^{*} \mathrm{sFr} 30,00$

ISBN 978-3-658-05649-0 (eBook)

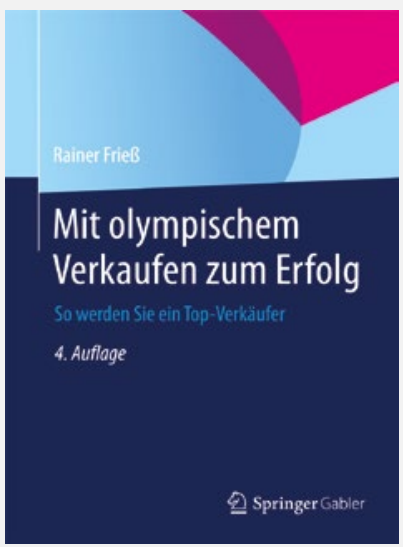

- Kompatktanleitung für die

Verkaufsgesprächsführung auf Spitzenniveau

- Kunden leichter überzeugen mit der passenden Verkaufsstrategie

- Mit kundentyp-adäquatem Verkaufen schneller ans (Verkaufs-)Ziel

$€$ (D) sind gebundene Ladenpreise in Deutschland und enthalten 7\% MwSt. $€$ (A) sind gebundene Ladenpreise in Österreich und enthalten 10\% MwSt. Die mit * gekennzeichneten Preise sind unverbindliche Preisempfehlungen und enthalten die landesübliche MwSt. Preisänderungen und Irrtümer vorbehalten. 